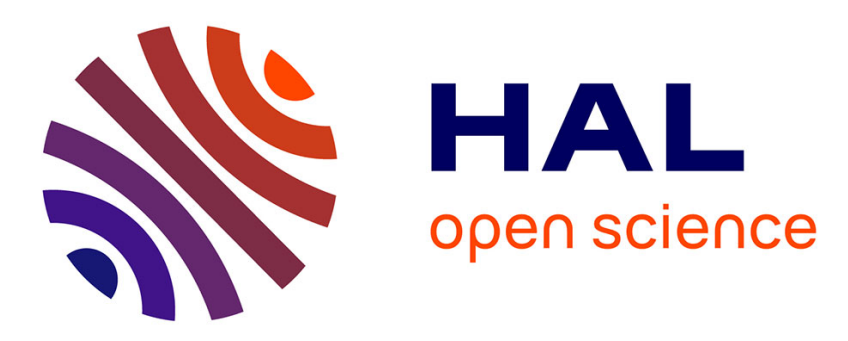

\title{
Relations sociales et ajustements à la crise : une analyse microstatistique comparative franco-britannique
}

\author{
Thomas Amossé, Philippe Askenazy, Martin Chevalier, Christine Erhel,
} Héloïse Petit, Antoine Reberioux

\section{- To cite this version:}

Thomas Amossé, Philippe Askenazy, Martin Chevalier, Christine Erhel, Héloïse Petit, et al.. Relations sociales et ajustements à la crise: une analyse microstatistique comparative franco-britannique. Revue internationale du travail, 2019, vol. 158 (n 3), pp. 509-535. 10.1111/ilrf.12092 . hal-02335728

\section{HAL Id: hal-02335728 \\ https://hal.univ-lille.fr/hal-02335728}

Submitted on 12 Nov 2019

HAL is a multi-disciplinary open access archive for the deposit and dissemination of scientific research documents, whether they are published or not. The documents may come from teaching and research institutions in France or abroad, or from public or private research centers.
L'archive ouverte pluridisciplinaire HAL, est destinée au dépôt et à la diffusion de documents scientifiques de niveau recherche, publiés ou non, émanant des établissements d'enseignement et de recherche français ou étrangers, des laboratoires publics ou privés. 


\title{
Relations sociales et ajustements à la crise: une analyse microstatistique comparative franco-britannique
}

\author{
Thomas AMOSSÉ*, Philippe ASKENAZY**, Martin CHEVALIER***, \\ Christine ERHEL****, Héloïse PETIT***** \\ et Antoine REBÉRIOUX******
}

\begin{abstract}
Résumé. Dans cette étude comparative sur la France et la Grande-Bretagne, les auteurs analysent les liens entre relations professionnelles et ajustements (des effectifs et des salaires) face à la crise de 2007-2008, en s'appuyant sur deux enquêtes au niveau des établissements, fortement comparables, l'une britannique (WERS), l'autre française (REPONSE), collectées en 2010-2012. Malgré des contextes différents (composition du tissu productif, temporalité et impact de la crise), les liens entre relations sociales et stratégies d'ajustement semblent proches (la présence syndicale ne suffisant pas à empêcher les ajustements). La différenciation des systèmes de relations professionnelles ne permet donc pas d'expliquer les divergences de modes d'ajustement constatées au niveau macroéconomique.
\end{abstract}

a France comme le Royaume-Uni ont été fortement touchés par la crise de 2007, avec un recul du produit intérieur brut (PIB) de 2,9 pour cent en 2009 en France ${ }^{1}$, et de 0,3 pour cent en 2008 et 4,2 pour cent en 2009 au

* Conservatoire national des arts et métiers (Cnam), Laboratoire interdisciplinaire pour la sociologie économique (Lise), Centre d'études de l'emploi et du travail (CEET); thomas.amosse@ lecnam.net. $\quad * *$ Centre Maurice Halbwachs $(\mathrm{CMH})$, École normale supérieure (ENS), Centre national de la recherche scientifique (CNRS); philippe.askenazy@ens.fr. *** Institut national de la statistique et des études économiques (INSEE); martin.chevalier@insee.fr. **** Cnam, Laboratoire interdisciplinaire de recherches en sciences de l'action (Lirsa), CEET; christine.erhel@lecnam.net (auteure de correspondance). $* * * * *$ Université de Lille, Centre lillois d'études et de recherches sociologiques et économiques (Clersé), CEET; heloise.petit@univ-lille.fr. ****** Université Paris-Diderot - Paris 7, laboratoire Dynamiques sociales et recomposition des espaces (LADYSS); antoine.reberioux@gmail.com. Cette recherche a bénéficié d'un financement de la Direction de l'animation de la recherche, des études et des statistiques (Dares) du ministère français du Travail, dans le cadre de l'exploitation de l'enquête REPONSE. Les auteurs remercient par ailleurs la Dares pour l'accès aux données de l'enquête REPONSE et le UK Data Archive, organisme britannique de gestion des données de recherche en sciences sociales, pour l'accès aux données de l'enquête WERS. Ils remercient également John Forth de son aide dans l'utilisation de l'enquête WERS.

Les articles paraissant dans la Revue internationale du Travail n'engagent que leurs auteurs, et leur publication ne signifie pas que le BIT souscrit aux opinions qui y sont exprimées.

${ }^{1}$ Il s'agit de la croissance du PIB réel en volume. En 2008, cet indicateur a crû faiblement (de 0,3 pour cent) en France.

Droits réservés (C) auteur(s), 2019.

Compilation des articles (C) Organisation internationale du Travail, 2019. 
Royaume-Uni, selon les données d'Eurostat. Cette dégradation de l'activité économique s'est accompagnée d'une nette hausse du taux de chômage (au sens du Bureau international du Travail) dans les deux pays, celui-ci étant passé de 7,7 pour cent en 2007 à 8,7 pour cent en 2009 en France, et de 5,3 pour cent en 2007 à 7,6 pour cent en 2009 au Royaume-Uni, toujours selon Eurostat. Les entreprises qui ont survécu y sont souvent parvenues au prix d'ajustements importants, de leur production bien sûr, mais aussi de leur organisation et de leur force de travail. Le choix des modalités d'ajustement se fait en relation avec de multiples facteurs, tels que la structure capitalistique et la taille de l'entreprise, le degré d'exposition à la crise du marché sur lequel elle opère et le type de relations sociales en son sein. Par sa simultanéité et son ampleur, la Grande Récession offre une expérience naturelle remarquable pour étudier plus avant le rôle des relations sociales dans la différenciation des pratiques d'ajustement des entreprises selon les pays. C'est l'objectif de cet article.

Si l'on adopte le cadre analytique des «variétés du capitalisme» (Hall et Soskice, 2001; Amable, 2003), on peut s'attendre à des réactions différentes des économies française et britannique à la Grande Récession. En effet, suivant cette approche, le modèle «libéral» britannique s'oppose au capitalisme «coordonné» à la française. Sur le marché du travail, cette opposition se traduit par une régulation forte côté français, impliquant l'État et des partenaires sociaux structurés, tandis qu'en Grande-Bretagne ${ }^{2}$ les stratégies individuelles dominent, avec une plus faible coordination des acteurs. Si l'on fait l'hypothèse que l'intensité des relations sociales constitue un frein à la mise en œuvre d'ajustements, notamment par les salaires, touchant le noyau des salariés stables, on peut s'attendre à ce que ce type d'ajustements ait été moins fréquent en France qu'en Grande-Bretagne.

Pour l'heure, quelques divergences entre les deux pays semblent apparaître, même si la comparaison est difficile du fait de différences de calendrier et d'intensité de la crise. Ainsi, il est établi, au niveau macroéconomique, que la Grande-Bretagne a connu un niveau de flexibilité interne (par les salaires) et externe (par l'emploi) relativement important (van Wanrooy et coll., 2013; Bryson et Forth, 2016): les salaires réels ont diminué dans un contexte d'inflation élevée ${ }^{3}$ et la durée du travail a baissé ${ }^{4}$ alors que l'emploi total était également affecté; en France, si les ajustements par l'emploi ont été importants, ils se sont focalisés sur l'emploi temporaire. On peut a priori lire ces observations

${ }^{2}$ Nous signalons que nous nous concentrons dans notre étude sur la situation en GrandeBretagne (plutôt qu'au Royaume-Uni dans son ensemble). En effet, les données de l'enquête WERS que nous utilisons ne sont pas collectées sur la totalité du territoire du Royaume-Uni, mais en Grande-Bretagne uniquement (elles portent sur l'Angleterre, l'Écosse et le Pays de Galles, mais pas sur l'Irlande du Nord).

${ }^{3}$ Selon Eurostat, l'indice des prix à la consommation harmonisés a progressé en France de 3,2 pour cent en 2008 , puis 0,1 pour cent en 2009 et 1,7 pour cent en 2010 , contre 3,6 pour cent, puis 2,2 pour cent, et ensuite 3,3 pour cent au Royaume-Uni. Le différentiel s'est encore accru en 2011 avec respectivement 2,3 et 4,6 pour cent.

${ }^{4}$ Selon les données de l'OCDE, la durée annuelle moyenne du travail passe de 1677 heures en 2007 à 1625 heures en 2011. 
comme une confirmation de l'opposition entre deux modèles de capitalisme divergents: le poids des relations sociales dans un capitalisme «coordonné» à la française expliquerait le moindre recours aux formes d'ajustements pesant sur le noyau des salariés stables alors que le modèle «libéral» britannique expliquerait l'utilisation de modes d'ajustements multiples. Toutefois, sur données agrégées, il est impossible d'identifier précisément la diversité des pratiques des entreprises et des mécanismes à l'œuvre: des caractéristiques saillantes au niveau national peuvent être le fait d'établissements différents.

Nous proposons de recourir à des données à l'échelon des établissements afin de mener une analyse fine des modes de gestion de l'emploi face à la crise, en soulignant leur diversité, entre pays mais également entre établissements. C'est ainsi au niveau de l'établissement que nous pourrons mettre en regard les relations sociales et les pratiques d'ajustement face à la crise. Cela paraît d'autant plus nécessaire que l'opposition entre les institutions et le mode de fonctionnement du marché du travail en France et en Grande-Bretagne ne doit pas être durcie à l'excès. En ce qui concerne le pouvoir et la place des syndicats, une analyse comparée des relations professionnelles (Crouch, 1993; Meardi, 2012) souligne la similitude de la France et de la Grande-Bretagne par leur rejet du corporatisme, une place limitée de la négociation collective et un pouvoir relativement faible des syndicats. En matière de gestion de l'emploi, l'étude de Caroli et ses coauteurs (2010), fondée sur une série de monographies d'entreprise conduites en Grande-Bretagne et en France dans le secteur agroalimentaire, souligne qu'une opposition trop schématique entre les deux pays n'est pas toujours juste: en particulier, le degré réel de flexibilité du travail serait aussi élevé des deux côtés de la Manche. Outre ces éléments de rapprochement entre les deux économies, la diversité des systèmes d'emploi à l'intérieur des pays, et même des secteurs d'activité (Marsden, 2010; Amossé et coll., 2016), peut également faire douter de la pertinence d'une analyse comparative strictement assise sur le paradigme des variétés du capitalisme.

Dans cet article, suivant une perspective microstatistique et comparative, nous étudions donc le lien existant entre la présence syndicale, et plus généralement les relations sociales, et l'évolution de l'emploi et des salaires dans les établissements français et britanniques à la suite de la crise de 2007. Pour ce faire, nous utilisons deux enquêtes très similaires, dont la méthodologie comme les questionnaires sont proches: l'enquête REPONSE (Relations professionnelles et négociations d'entreprise), dans son édition 2010-2011, et l'enquête WERS 2011 (ou WERS 6), sixième vague de la Workplace Employment Relations Study, étude sur les relations professionnelles en Grande-Bretagne, pour laquelle la collecte des données a eu lieu en 2011-2012. Notre article se situe dans le champ de l'analyse comparative de la relation d'emploi (Bryson et Frege, 2010), et s'inscrit dans la lignée d'autres travaux ayant déjà utilisé de façon conjointe les enquêtes REPONSE et WERS ${ }^{5}$.

\footnotetext{
${ }^{5}$ Voir en particulier Coutrot, 1998; Caroli et Van Reenen, 2001; Conway et coll., 2008; Marsden et Belfield, 2010; Bryson, Forth et Laroche, 2011; Marsden, 2013; et Amossé et coll., 2016.
} 
Il ressort des investigations empiriques conduites que, dans les deux pays, les établissements ayant procédé à des ajustements suite à la Grande Récession ne sont pas moins syndiqués que les autres, toutes choses égales par ailleurs. Mais, parce qu'elles résultent de mécanismes complexes, les associations mises en évidence se présentent sous la forme de corrélations contrôlées et ne permettent pas, en tant que telles, d'identifier des causalités. Aussi différents indicateurs caractérisant les relations sociales à l'échelon de l'établissement sont-ils utilisés afin de proposer des pistes d'interprétation: l'existence de négociations sur l'emploi ou les salaires, la présence de conflits collectifs et enfin l'opinion des directions d'entreprise quant à la présence syndicale. Il en découle notamment que, si les opinions managériales favorables à l'expression des salariés sont négativement associées à une diminution des effectifs ou des salaires à l'occasion de la crise, ce n'est pas le cas de la présence syndicale, qui accompagne la mise en œuvre de ces ajustements.

Nous organisons la suite de notre article en quatre parties. La première est consacrée à la discussion des éléments de contexte sur les relations sociales en France et en Grande-Bretagne, notamment pendant la crise. Dans notre deuxième partie, nous présentons les données utilisées et nous décrivons les pratiques d'ajustement de l'emploi ou des salaires au sein des établissements français et britanniques de l'échantillon. Dans une troisième partie, nous examinons ces pratiques d'ajustement à la lumière des profils des établissements en matière de relations sociales, notamment de la présence syndicale, sur la base d'un ensemble de régressions logistiques. Nous consacrons une quatrième et dernière partie à notre conclusion.

\section{Les relations sociales face à la crise: éléments de contexte}

\section{Les relations sociales en France et en Grande-Bretagne: des tendances communes malgré des traditions différentes}

Les systèmes de relations professionnelles français et britanniques sont institués selon des logiques propres que l'on peut identifier en termes géographiques, le système britannique situant le niveau local en son centre alors que le niveau national reste une référence prégnante en France (Crouch, 1993; Meardi, 2012; Milner, 2015; Amossé et Forth, 2016). En Grande-Bretagne, la capacité d'influence des syndicats découle directement de leur capacité à mobiliser les salariés localement. Si un syndicat peut toujours nommer un représentant dans un établissement, la signature d'un «accord de reconnaissance ${ }^{6}$ est la condition sine qua non de l'ouverture de négociations avec les représentants syndicaux sur les conditions de travail et d'emploi (et notamment les salaires). Le pouvoir et le financement des syndicats dépendent directement de

${ }^{6}$ Depuis 1999, pour les établissements de plus de 20 salariés, cette reconnaissance est obligatoire si au moins 50 pour cent des salariés sont syndiqués. 
leur aptitude à recruter des membres. En France, traditionnellement, la place et le rôle des syndicats sont d'abord définis au niveau national (Jobert, 2000; Bevort et Jobert, 2011). La négociation dans l'entreprise est un droit inscrit dans le Code du travail (pour les établissements de plus de 50 salariés), au même titre que le droit à l'information et à la consultation. Et ces négociations s'inscrivent dans le cadre de négociations nationales et de branche dans un système multiniveaux ${ }^{7}$. S'ajoute à cela le rôle donné aux syndicats dans la gestion paritaire d'institutions nationales telles que l'Unédic ou la Sécurité sociale par exemple (organismes gérant respectivement les allocations chômage et les cotisations sociales). L'approche britannique se place donc dans une logique de «laissez-faire» décentralisé qui contraste avec l'approche française où les syndicats ont une légitimité acquise au niveau national, même lorsqu'ils sont peu présents localement.

Malgré ces différences structurelles, les deux pays ont connu au cours des dernières décennies des tendances communes vers une moindre syndicalisation et, depuis les années 1980, vers la décentralisation des négociations. En France, on assiste à une recomposition des espaces de dialogue social dont la progression continue des négociations collectives d'entreprise est le fait saillant (Bloch-London et Pélisse, 2008; Bevort et Jobert, 2011; Jobert, 2017). Le cadre législatif a introduit l'obligation de négocier au niveau local en 1982, avec les lois Auroux, puis l'a appliquée à un nombre croissant de domaines. Ces obligations de plus en plus nombreuses se sont accompagnées de dérogations de plus en plus fréquentes à la «hiérarchie des normes» (voir note 7), ce qui a progressivement permis aux négociations d'entreprise de s'affranchir, du moins partiellement, des négociations engagées à un niveau supérieur (voir la loi de 2004 relative à la formation professionnelle tout au long de la vie et au dialogue social et, pour le temps de travail, la loi de 2008 portant rénovation de la démocratie sociale et réforme du temps de travail). Dans le même temps, la branche comme le niveau interprofessionnel, bien qu'actifs, ont vu leur légitimité s'éroder. Si la quasi-totalité des salariés français restent couverts par une convention collective négociée au niveau de la branche, cet espace de négociation fait l'objet de critiques appuyées de la part du patronat, ce qui laisse craindre la multiplication d'accords ne protégeant qu'au minimum les salariés à ce niveau. La négociation interprofessionnelle quant à elle est l'objet d'une attention croissante de la part des pouvoirs publics, qui en ont même fait un préalable à toute réforme portant sur le travail, l'emploi et la formation (loi de modernisation du dialogue social de 2007). Si de tels accords sont effectivement signés, la place centrale ainsi dévolue à l'échelon interprofessionnel a également mis en lumière des blocages et désaccords entre partenaires sociaux et contribué à remettre en question leur légitimité d'intervention à ce

\footnotetext{
7 Jusqu'aux réformes du Code du travail de 2016 et 2017, l'articulation entre les niveaux de négociation était régie par le «principe de faveur» qui stipulait qu'un texte négocié à un niveau inférieur ne pouvait modifier un texte supérieur qu'à condition de l'améliorer.
} 
niveau. Sans occulter le rôle fondamental que gardent les branches et le niveau interprofessionnel, on peut conclure de ces transformations que l'entreprise a progressivement pris une place centrale dans le système de relations professionnelles français.

Malgré un contexte institutionnel bien différent, la dynamique des relations professionnelles britanniques s'illustre également par une focalisation croissante sur la négociation à l'échelon de l'entreprise (Marginson, 2015; Brown, Bryson et Forth, 2009). Les années 1980 ont d'abord vu l'effondrement des négociations multiemployeurs. Au départ centrales, ces négociations sont devenues marginales. La décentralisation des négociations n'est pas seulement la contrepartie de cette perte de vitesse puisqu'elle l'a précédée et continue aujourd'hui. Plutôt qu'à un mouvement de simple décentralisation, on assiste à une centralité croissante de l'entreprise, car on constate également une consolidation des négociations à ce niveau, qui portent désormais sur l'ensemble des catégories de salariés alors qu'elles faisaient traditionnellement l'objet de négociations différenciées. Ces transformations sont le fruit des stratégies des acteurs, et en particulier des employeurs, combinées à des réformes législatives dont les deux axes principaux ont été les restrictions portées aux actions collectives, jusqu'en 1997, puis une préférence pour l'adossement de nouveaux droits sociaux au cadre législatif plutôt que négocié (Dickens et Hall, 2003).

Un autre point commun des dynamiques des systèmes français et britannique au cours des dernières années est le poids croissant des accords portant sur l'emploi et la compétitivité (Marginson, 2015; Jobert, 2017). Cette tendance est évidemment au cœur des négociations postcrise.

\section{Quelle place pour les relations sociales face à la Grande Récession?}

Au niveau national, l'analyse comparative des politiques économiques souligne l'existence d'un consensus entre les partenaires sociaux dans la plupart des pays sur un objectif de préservation de l'emploi, y compris par un dialogue social innovant portant sur l'emploi et la compétitivité (Commission européenne, 2011; Freyssinet, 2016). En France, la collaboration entre les partenaires sociaux et le gouvernement a été relancée dès le début de la crise, malgré des tensions entre syndicats et organisations d'employeurs. Deux sommets sociaux ont été organisés par le gouvernement en 2008 et 2009, et un Fonds d'investissement social (Fiso) a été installé en avril 2009 afin de coordonner les actions de l'État et des partenaires sociaux. En parallèle, des accords nationaux interprofessionnels ont été négociés sur l'indemnisation du chômage partiel et le renforcement de dispositifs de reclassement (contrats de reclassement et de transition professionnels). En Grande-Bretagne, les désaccords entre syndicats et organisations d'employeurs sur la réponse à apporter à la crise n'ont pas permis de promouvoir des mesures négociées (Commission européenne, 2011). Les syndicats soutenaient la mise en place d'un dispositif de chômage partiel, tandis que les employeurs réclamaient des mesures de licenciement 
temporaire et de soutien aux entreprises. Un sommet sur l'emploi a été organisé par le gouvernement en janvier 2009, mais n'a abouti à aucune mesure.

Les réponses à la crise ont ainsi reposé à la fois sur des négociations de pactes sectoriels ou nationaux pour la compétitivité et l'emploi et sur des négociations directement conduites au niveau des entreprises (Hyman, 2010). Ces tendances s'observent bien sûr dans le cas emblématique de l'Allemagne, où le système de relations professionnelles coopératif et, plus particulièrement, l'existence de pactes négociés entre partenaires sociaux ont permis le maintien des emplois et soutenu la compétitivité des entreprises (Bellmann, Gerner et Laible, 2016; Zagelmeyer, 2016). Mais elles concernent également d'autres pays et s'inscrivent dans le développement de négociations sur l'emploi et la compétitivité dans les entreprises, également appelées «négociations de compromis» (concession bargaining), dont la tendance est née bien avant la crise (Marginson, 2015; Roche, Teague et Coughlan, 2015; Távora et González, 2016; Freyssinet, 2016). Lors de ces négociations, les salariés font des concessions à l'employeur (souvent en termes de salaire) en échange d'un engagement de ce dernier au maintien de certaines conditions (habituellement en termes d'emploi). En période de crise, la multiplication de ce type de négociations a alimenté le phénomène de décentralisation des relations professionnelles (Glassner, Keune et Marginson, 2011; Béthoux et coll., 2015).

Dans ce contexte, notre article étudie les liens, au niveau des établissements, entre relations sociales et réaction à la Grande Récession en France et en Grande-Bretagne sur la base de données statistiques: quels ajustements ont eu lieu dans les établissements selon la place qu'y tiennent les relations sociales? Ne disposant pas des moyens d'étayer de façon stricte des liens de causalité, nous adoptons ici une démarche résolument descriptive. Si les travaux empiriques sont pour l'instant restés relativement rares, nous nous situons dans la lignée des travaux déjà effectués (van Wanrooy et coll., 2013; Amossé et coll., 2016) avec une triple originalité: 1) nous examinons les ajustements internes (par les salaires) et externes (par les effectifs) non pas séparément, mais en interaction; 2) nous adoptons une démarche comparative, en proposant des interprétations possibles des résultats en fonction des contextes institutionnels prévalant en France et en Grande-Bretagne; 3) enfin, nous utilisons non seulement des variables relatives à la présence syndicale, mais aussi des indicateurs permettant de caractériser plus largement les relations sociales dans l'établissement (négociation, conflit, opinions des directions d'entreprise).

\section{Données et stratégie économétrique}

\section{Les enquêtes WERS et REPONSE}

Notre analyse empirique s'appuie sur les données recueillies à l'échelon des établissements dans le cadre des enquêtes REPONSE 2010-2011 et WERS 6, menées quasi simultanément. Ces deux enquêtes «cousines» (Amossé et Coutrot, 2008) fournissent des informations particulièrement précises sur les 
relations professionnelles et les modalités d'ajustement face aux évolutions de l'activité économique. Les informations que nous utilisons ont été recueillies par le biais d'entretiens en face à face avec un membre de la direction, autant que possible un responsable des ressources humaines ${ }^{8}$.

Nous avons procédé à des estimations séparées sur les deux enquêtes. En effet, les différences de formulation de certaines questions et les spécificités institutionnelles rendent délicate l'utilisation directe des données agrégées. Un champ commun a été défini en ne retenant que les établissements de 11 salariés et plus du secteur marchand non agricole. Cette opération conduit à n'inclure que 1602 des 2680 observations de l'enquête WERS et 3947 des 4023 observations de l'enquête REPONSE.

Les enquêtes REPONSE et WERS contiennent chacune une variable pouvant renseigner sur le degré d'exposition de l'établissement à la crise. Dans le cas de WERS, il est directement demandé au représentant de la direction interviewé d'évaluer, pour l'établissement, l'ampleur du choc causé par la crise (sans précision de dates) $)^{9}$. Pour REPONSE, la question porte sur la dynamique du volume d'activité de l'établissement entre 2008 et $2010^{10}$. Ces deux variables sont endogènes (elles dépendent des réponses adoptées suite à la crise), et ne sont pas strictement comparables: la question britannique interroge certes plus directement sur les conséquences de la crise, mais elle est moins factuelle. Elles ne constituent donc pas des mesures précises de l'ampleur du choc. Nous faisons néanmoins l'hypothèse que toutes deux permettent de déterminer si l'établissement connaît une situation économique dégradée à la suite de la crise.

Afin d'identifier et de comparer les modalités d'ajustement dans les deux pays, nous avons construit deux indicateurs, l'un relatif aux salaires ou rémunérations ${ }^{11}$ (en valeur nominale), l'autre aux effectifs.

- Concernant les salaires, nous avons utilisé deux questions comparables, interrogeant les dirigeants sur l'impact de la crise. La variable de WERS permet d'identifier «le gel ou la baisse des salaires» opérés «pour faire face à la récente récession». Celle de REPONSE porte sur les «politiques de modération, le gel ou la baisse des rémunérations» pour «faire face à la crise».

- La construction d'un indicateur d'ajustement par l'emploi s'appuie, dans REPONSE, sur une question portant sur l'évolution des effectifs pour l'ensemble des salariés. Cette question, qui n'établit pas de lien avec la crise, distingue les situations de «hausse», de «baisse» ou de «stabilité» et porte sur les trois dernières années (2008-2010). Pour WERS, nous utilisons de

${ }^{8}$ Les traductions (respectivement en anglais et en français) de la documentation et des questionnaires des enquêtes REPONSE et WERS sont disponibles à l'adresse http://www.niesr.ac.uk/ projects/employment-relations-britain-and-france [consulté le 13 juin 2019].

${ }^{9}$ Impact «très important», «important», «modéré», «faible», «pas d'impact».

10 «Fortement croissant», «croissant», «stable», «décroissant», «fortement décroissant».

${ }^{11}$ Dans la suite de l'article, pour simplifier, nous n'utiliserons que le terme «salaire» et non «salaire ou rémunération». 
nouveau la question sur la réaction de l'établissement face à la récession pour repérer l'existence de départs involontaires (licenciements) ou volontaires (démissions), ou un moindre recours au personnel intérimaire ou temporaire.

Pour ce qui est des variables indicatrices des relations sociales (que nous décrivons plus précisément dans une présentation fournie en annexe), les informations recueillies à partir des enquêtes REPONSE et WERS permettent en premier lieu d'identifier la présence syndicale dans les établissements en 2011 (par le biais d'un représentant syndical ou d'au moins un salarié syndiqué sur le site $)^{12}$. Trois autres variables sont utilisées pour préciser la situation des relations sociales dans l'établissement, avec ou sans présence syndicale: l'existence de négociations ou de consultations sur l'emploi et les salaires, la survenue de conflits collectifs du travail au cours des trois dernières années (2008-2010) en France et la survenue ou la menace de tels conflits au cours des douze derniers mois en Grande-Bretagne, et enfin l'opinion des représentants de la direction à propos des syndicats. Ces variables ne sont pas strictement comparables, ce qui explique notamment pourquoi nous n'avons pas fusionné les bases française et britannique.

Les deux enquêtes fournissent par ailleurs des informations sur les caractéristiques structurelles des établissements, qui sont utilisées comme variables de contrôle dans nos estimations: le secteur d'activité (onze positions), l'ancienneté et la taille de l'établissement (en cinq et six tranches), l'indépendance de l'établissement (entendue comme la non-appartenance à une organisation plus large, que ce soit une entreprise ou un groupe), la cotation en bourse (directe ou indirecte, via le siège social ou la maison mère), la détention majoritaire du capital social par une famille et la dimension du marché (local, régional, national ou international).

Nous introduisons aussi parmi les variables de contrôle la structure de la main-d'œuvre au niveau de l'établissement (proportion de femmes; de salariés de 21 ans ou moins et de 50 ans ou plus; de cadres et d'ingénieurs, de techniciens et d'agents de maîtrise, d'employés et d'ouvriers; de contrats à durée déterminée (CDD), d'intérimaires).

\section{Les profils des établissements français et britanniques et l'impact de la crise}

Les statistiques descriptives sur les données de REPONSE et de WERS font apparaître des différences importantes entre les tissus productifs britanniques et français ${ }^{13}$. En Grande-Bretagne, les établissements sont relativement plus

12 Cette définition large vise à identifier des espaces, désormais réduits dans le secteur marchand en Grande-Bretagne, où les syndicats existent, même de façon minimale (pour une comparaison approfondie des modalités de présence syndicale dans les deux pays, voir Amossé et Forth, 2016). Des analyses ont été conduites avec des définitions plus restrictives (présence d'un représentant syndical dans l'établissement et/ou syndicat reconnu par la direction pour négocier). Elles donnent des résultats convergents (obtention possible sur demande auprès des auteurs).

13 L'ensemble des statistiques mobilisées sont pondérées, c'est-à-dire représentatives des établissements de 11 salariés ou plus du secteur marchand non agricole. Elles sont présentées en annexe (voir tableau A1). 
jeunes, et de plus petite taille, comparativement à la France. La Grande-Bretagne se distingue par une proportion plus élevée d'établissements et de salariés dans le secteur de l'hébergement et de la restauration et celui des services spécialisés, scientifiques et techniques, ainsi que dans les activités financières, d'assurance et immobilières, ou encore dans le secteur de l'information, de la communication et des spectacles. A contrario, la part des établissements de l'industrie, de la construction et des transports est nettement plus faible qu'en France. Du point de vue de l'exposition à la concurrence, on relève que la France comporte un plus grand nombre d'établissements situés sur un marché international. Reste que les relations capitalistiques apparaissent assez proches de part et d'autre de la Manche, en ce qui concerne tant l'autonomie des établissements que la propension à être coté en Bourse.

Les différences de structures institutionnelles et juridiques entre les deux pays se traduisent par des profils de relations sociales divergents (tableau 1). La présence syndicale (au moins un représentant syndical ou membre syndiqué dans l'établissement) est bien plus importante en France, où elle concerne 41 pour cent des établissements (contre 21,7 pour cent en GrandeBretagne). Pour les indicateurs complémentaires de relations sociales, malgré des différences de définition dans les deux enquêtes, les données semblent montrer qu'il y a davantage de négociations et/ou de consultations en France sur les salaires et l'emploi, et une plus grande conflictualité dans les établissements français. L'indicateur construit quant à l'opinion des dirigeants sur la syndicalisation ou le rôle des syndicats permet d'identifier dans les deux pays une proportion similaire (environ 9 pour cent) d'établissements «prosyndicaux».

Concernant l'exposition à la crise, près de la moitié des établissements britanniques déclarent être «très fortement» ou «fortement affectés» (tableau 1). En France, ils sont moitié moins à déclarer une activité à la baisse. Ces proportions ne peuvent être comparées directement - les questions étant de nature différente dans les deux enquêtes, comme mentionné plus haut -, mais elles sont cohérentes avec l'ampleur du choc macroéconomique, qui est plus prononcé en Grande-Bretagne. Parmi les établissements qui se déclarent affectés par la crise, le cas britannique se distingue par une plus forte proportion de petits établissements (11 à 19 salariés), d'établissements autonomes ${ }^{14}$, ou encore d'établissements du secteur financier. En France, les établissements déclarant une activité à la baisse se situent plus souvent dans les secteurs de l'industrie, de la construction et du commerce et sont davantage implantés sur un marché international.

En lien avec les différences de régulation et de fonctionnement du marché du travail, on s'attend à des réactions différentes des établissements face à la crise. Les données permettent de le vérifier (tableau 2): bien que les variables utilisées ne soient pas rigoureusement comparables, les établissements britanniques ont, semble-t-il, plus souvent ajusté leur niveau d'emploi (30,6 pour cent,

${ }^{14}$ C'est-à-dire qui n'appartient pas à un groupe ou à une entreprise multiétablissements. 


\section{Tableau 1. Comparaison des dynamiques d'activité et des relations sociales dans les établissements français et britanniques (en pourcentage des établissements de l'échantillon)}

\begin{tabular}{|c|c|c|}
\hline & France & Grande-Bretagne \\
\hline \multicolumn{3}{|l|}{ Dynamique de l'activité/ampleur de la crise } \\
\hline Stable ou à la hausse/pas ou peu affecté & 75,8 & 53,2 \\
\hline À la baisse/très fortement ou fortement affecté & 24,2 & 46,8 \\
\hline \multicolumn{3}{|l|}{ Relations sociales } \\
\hline Présence d'un représentant syndical ou d'au moins un salarié syndiqué & 41,0 & 21,7 \\
\hline $\begin{array}{l}\text { Négociation sur l'emploi (2008-2010)/consultation sur des licenciements } \\
\text { économiques envisagés au cours des douze derniers mois }\end{array}$ & 39,2 & 16,9 \\
\hline $\begin{array}{l}\text { Négociation sur les salaires (2008-2010)/négociation ou consultation } \\
\text { lors de la dernière révision salariale }\end{array}$ & 53,6 & 8,9 \\
\hline $\begin{array}{l}\text { Conflit (2008-2010)/conflits ou menace de conflit au cours } \\
\text { des douze derniers mois }\end{array}$ & 17,0 & 3,3 \\
\hline Syndicats irremplaçables/en faveur de la syndicalisation des salariés & 8,9 & 9,1 \\
\hline
\end{tabular}

Tableau 2. Modes d'ajustement à la baisse de l'emploi et des salaires (en pourcentage des établissements de l'échantillon)

\begin{tabular}{|c|c|c|}
\hline & France & Grande-Bretagne \\
\hline \multicolumn{3}{|l|}{ Emploi } \\
\hline \multicolumn{3}{|l|}{$\begin{array}{l}\text { Pas de diminution/ni licenciements, ni départs volontaires, } \\
\text { ni moindre recours aux emplois temporaires }\end{array}$} \\
\hline \multicolumn{3}{|l|}{ Diminution/licenciements, départs volontaires ou moindre recours } \\
\hline \multicolumn{3}{|l|}{ Salaire } \\
\hline Pas d'impact connu de la crise sur les salaires* & 60,5 & 61,1 \\
\hline Modération & 27,4 & Non posé \\
\hline Gel ou baisse & 12,2 & 38,9 \\
\hline Ajustements emploi et/ou salaire, dont & 25,8 & 53,3 \\
\hline Ajustement à la baisse de l'emploi uniquement & 13,7 & 13,4 \\
\hline Ajustement à la baisse des salaires** uniquement & 7,3 & 21,7 \\
\hline Ajustement à la baisse de l'emploi et des salaires & 4,8 & 17,2 \\
\hline \multicolumn{3}{|c|}{$\begin{array}{l}\text { * La modération salariale n'étant pas posée dans WERS, les deux pourcentages de la première ligne de la partie «sa- } \\
\text { laire» ne sont pas comparables terme à terme (la valeur présentée pour la Grande-Bretagne pouvant inclure des situa- } \\
\text { tions de modération salariale). ** Variable fondée uniquement sur la réponse "gel ou baisse», qui est comparable dans } \\
\text { les deux pays. }\end{array}$} \\
\hline
\end{tabular}


contre 18,5 pour cent en France) $)^{15}$ et pratiqué des gels ou des baisses de salaire (38,9 pour cent, contre 12,2 pour cent en France). Cet écart se retrouve lorsque l'on combine les deux types de flexibilité pris en compte ici: l'absence d'ajustement caractérise une très large majorité d'établissements français $(74,2$ pour cent, contre 47,7 pour cent en Grande-Bretagne). Par ailleurs, l'orientation (interne ou externe) des ajustements diffère dans les deux pays: ils sont davantage orientés vers les salaires en Grande-Bretagne et vers l'emploi en France. Ces résultats descriptifs confirment la plus forte flexibilité des établissements britanniques, notamment sur le plan salarial, en accord avec les approches de type «variétés du capitalisme». Ils sont également en cohérence avec la priorité donnée à l'emploi par les syndicats britanniques (Delahaie et Vincent, 2016). Mais ces comparaisons en niveau sont à prendre avec prudence étant donné les différences entre les questionnaires, surtout concernant l'évolution de l'emploi, et les effets de composition liés aux différences de structure sectorielle des deux pays. Ces réticences sont largement levées dès lors qu'on ne s'intéresse plus à l'écart de niveau de flexibilité entre la France et la Grande-Bretagne, mais - comme nous allons le faire - aux caractéristiques «toutes choses égales par ailleurs» des établissements ayant ajusté l'emploi et/ou les salaires dans chaque pays.

\section{Stratégie économétrique}

Tout en revendiquant une logique descriptive, compte tenu des multiples sources d'hétérogénéité au sein de nos données, une analyse économétrique est nécessaire pour cerner les mécanismes sous-jacents. Un ensemble de régressions logistiques ont été estimées pour chacun des pays. Des modèles binomiaux ont permis d'établir la situation sociale «toutes choses égales par ailleurs» (c'està-dire à choc économique et caractéristiques structurelles donnés) des établissements ayant procédé à des ajustements, que ce soit de l'emploi et/ou des salaires. L'analyse porte tout d'abord sur la seule présence syndicale (modèle I), puis est étendue aux autres indicateurs des relations sociales (modèle II) et enfin ajoute des termes d'interaction avec la présence syndicale (modèle III). Des modèles multinomiaux non ordonnés ont ensuite été estimés afin de préciser, parmi les établissements ayant procédé à des ajustements, le profil des relations sociales de ceux qui ont opté pour des ajustements externes (par l'emploi, seul ou de façon jointe avec les salaires) plutôt qu'uniquement internes (par les seuls salaires): le modèle IV reprend le même ensemble de variables que le modèle I, limitant l'analyse des relations sociales à la présence syndicale; le modèle $\mathrm{V}$ intègre, comme le modèle II, l'ensemble des variables indicatrices des relations sociales (sans termes d'interaction). Un sixième modèle suivant une spécification analogue au modèle III a été estimé, mais il n'est pas présenté dans l'article du fait de l'absence presque totale de coefficients significativement non nuls ${ }^{16}$.

${ }^{15}$ Ces ajustements ont pu prendre la forme de licenciements ou de ruptures de contrats, mais aussi de non-remplacements des départs en retraite et de fins de contrats à durée déterminée, ou encore d'un moindre recours à l'intérim.

${ }^{16}$ Cela s'explique par la réduction du nombre d'observations dans l'analyse des modes d'ajustement et la multiplication du nombre de variables avec les termes d'interaction. 
Tableau 3. Modes d'ajustement à la baisse selon la présence syndicale (en pourcentage des établissements de l'échantillon)

\begin{tabular}{|c|c|c|c|c|}
\hline & \multicolumn{2}{|c|}{ Présence syndicale* } & \multicolumn{2}{|c|}{ Pas de présence syndicale } \\
\hline & France & Grande-Bretagne & France & Grande-Bretagne \\
\hline \multirow{4}{*}{$\begin{array}{l}\text { Ajustements emploi et/ou salaire, dont } \\
\text { Ajustement à la baisse de l'emploi uniquement } \\
\text { Ajustement à la baisse des salaires** uniquement } \\
\text { Ajustement à la baisse de l'emploi et des salaires }\end{array}$} & 30,2 & 61,7 & 22,7 & 49,7 \\
\hline & 18,2 & 19,2 & 10,6 & 11,8 \\
\hline & 6,3 & 24,8 & 8,0 & 20,8 \\
\hline & 5,7 & 17,7 & 4,2 & 17,1 \\
\hline \multicolumn{5}{|c|}{$\begin{array}{l}\text { * La présence syndicale est définie par la présence d'un représentant syndical ou d'au moins un salarié syndiqué. ** En } \\
\text { ne retenant que la réponse "gel ou baisse", qui est comparable dans les deux pays. }\end{array}$} \\
\hline \multirow{2}{*}{\multicolumn{5}{|c|}{$\begin{array}{l}\text { Champ: Établissements de } 11 \text { salariés et plus du secteur marchand non agricole. } \\
\text { Note: Données pondérées (poids établissement). }\end{array}$}} \\
\hline & & & & \\
\hline \multicolumn{5}{|c|}{ Source: Calculs des auteurs à partir des données des enquêtes REPONSE 2010-2011 et WERS 2011.} \\
\hline
\end{tabular}

\section{Résultats: quels liens avec les relations sociales? Statistiques descriptives et estimations économétriques}

La présence syndicale demeure fortement structurante de la situation sociale dans l'établissement de part et d'autre de la Manche (Amossé et Forth, 2016). Sans prise en compte des effets de composition, le lien entre représentation syndicale et ajustements de l'emploi et des salaires semble proche entre les deux pays (tableau 3). Dans les deux pays, la présence syndicale est associée à une fréquence supérieure des ajustements. L'association est particulièrement marquée pour les ajustements par l'emploi uniquement. Une exception apparaît pour les ajustements par les salaires uniquement en France, que l'on observe plus fréquemment dans les établissements non syndiqués.

Ces résultats pourraient refléter des différences d'implantation syndicale dans les secteurs d'activité et les entreprises selon le degré d'exposition à la crise. Les analyses qui suivent, basées sur des régressions logistiques, montrent qu'il n'en est rien. Elles confirment ces premiers éléments en permettant de raisonner, dans chacun des pays, à choc économique et caractéristiques structurelles des établissements donnés.

\section{Le profil «toutes choses égales par ailleurs» des relations sociales dans les établissements ayant opéré des ajustements}

Si l'on compare les relations sociales dans les établissements ayant procédé à des ajustements en France et en Grande-Bretagne, c'est la similitude qui frappe (tableau 4). Dans les deux pays, à choc économique et caractéristiques structurelles donnés, les établissements ayant réduit leurs effectifs et/ou leurs salaires dans le contexte de crise sont plus souvent syndiqués. Bien qu'il ne puisse être interprété de façon causale, ce résultat remet en cause l'idée selon laquelle l'action des syndicats empêcherait les ajustements en temps de crise, et ce, en France comme en Grande-Bretagne, malgré les spécificités institutionnelles nationales. 
Tableau 4. Variables relatives aux relations sociales, avec et sans interaction avec la présence syndicale, associées à un ajustement de l'emploi et/ou des salaires (modèles I, II et III)

\begin{tabular}{|c|c|c|}
\hline $\begin{array}{l}\text { Odds ratios issus des régressions logistiques binomiales } \\
\text { (situation de référence: absence d'ajustement à la baisse) }\end{array}$ & France & Grande-Br \\
\hline \multicolumn{3}{|l|}{ Modèle I, présence syndicale (et variables de contrôle) } \\
\hline Présence syndicale & $1,49 * * *$ & $1,31 *$ \\
\hline \multicolumn{3}{|c|}{$\begin{array}{l}\text { Modèle II, présence syndicale et autres variables relatives aux relations sociales, } \\
\text { sans interaction (et variables de contrôle) }\end{array}$} \\
\hline Présence syndicale & $1,35 * * *$ & $1,37 *$ \\
\hline Négociation/consultation sur l'emploi & $1,29 * * *$ & $3,10 * * *$ \\
\hline Négociation/négociation ou consultation sur les salaires & 0,95 & 0,69 \\
\hline Conflit/conflits ou menace de conflit & $1,50 * * *$ & 1,74 \\
\hline Syndicats irremplaçables/en faveur de la syndicalisation des salariés & $0,76 *$ & $0,58 * *$ \\
\hline
\end{tabular}

Modèle III, présence syndicale et autres variables relatives aux relations sociales, avec interaction (et variables de contrôle)

En présence de syndicat

Négociation/consultation sur l'emploi

Négociation/négociation ou consultation sur les salaires

$1,22 * 5,92 * * *$

Conflit/conflits ou menace de conflit

$1,12 \quad 0,80$

Syndicats irremplaçables/en faveur de la syndicalisation des salariés

$1,62 * * * 1,73$

En l'absence de syndicat

Négociation/consultation sur l'emploi

Négociation/négociation ou consultation sur les salaires

$0,72 * 0,69$

Conflit/conflits ou menace de conflit

$1,36 * * 2,58 * * *$

Syndicats irremplaçables/en faveur de la syndicalisation des salariés

$1,01 \quad 0,50$

$1,28 \quad 1,82$

$0,83 \quad 0,29 * * *$

* Statistiquement significatif au seuil de 10 pour cent. ** Statistiquement significatif au seuil de 5 pour cent.

*** Statistiquement significatif au seuil de 1 pour cent.

Champ: Établissements de 11 salariés et plus du secteur marchand non agricole.

Note: Données pondérées (poids établissement). Modèles: régressions logistiques binomiales avec, comme variable dépendante, les modes d'ajustement à la baisse de l'emploi et/ou des salaires (la situation de référence étant l'absence d'ajustement à la baisse) et, comme variables indépendantes, outre les variables relatives aux relations sociales, la variable rendant compte d'un choc économique et des variables de contrôles relatives aux caractéristiques structurelles (non indiquées dans le tableau, mais l'ensemble des résultats est disponible sur demande auprès des auteurs).

Source: Calculs des auteurs à partir des données des enquêtes REPONSE 2010-2011 et WERS 2011.

L'intégration d'autres variables relatives aux relations sociales permet de préciser l'interprétation (tableau 4). Ces analyses montrent en premier lieu que l'odds ratio relatif à la présence syndicale ${ }^{17}$ n'est que peu modifié (en comparaison avec les modèles I et II), confirmant l'association positive entre cette présence et l'existence d'ajustements, indépendamment des autres

${ }^{17}$ L'odds ratio (rapport des cotes) s'interprète comme la probabilité que l'établissement ait procédé à un ajustement (par l'emploi ou par les salaires), plutôt qu'il ne l'ait pas fait, quand un représentant syndical ou des salariés syndiqués sont présents en son sein plutôt qu'absents. Ce rapport de probabilité est toujours significativement supérieur à 1, en France comme en Grande-Bretagne, atteignant 1,35 et 1,37, respectivement, dans le modèle II (contre 1,49 et 1,31 dans le modèle I). 
caractéristiques des relations sociales (négociation ou consultation sur l'emploi ou les salaires, conflit collectif et opinions managériales à l'égard des syndicats). Le modèle II montre par ailleurs nettement la ressemblance en matière de situation sociale des établissements français et britanniques ayant procédé à des ajustements: des négociations ou consultations sur l'emploi y ont plus souvent eu lieu, de même que des conflits collectifs ${ }^{18}$, et les directions s'y déclarent moins souvent prosyndicales. L'absence de lien significatif avec des négociations sur les salaires est à noter. Elle peut être interprétée, nous le verrons, comme le signe d'ajustements sur les salaires qui se passent plus souvent de manière discrète (c'est-à-dire hors négociation) dans les entreprises: même si cette information n'est pas directement disponible, on peut supposer que les ajustements salariaux portent alors sur les parts variables des rémunérations.

Globalement, il semble que les ajustements opérés à la suite de la crise soient plus souvent le fait, en France comme en Grande-Bretagne, d'établissements où existent des possibilités d'expression collective, qui prennent la forme d'une présence institutionnelle (de représentants syndicaux ou de salariés syndiqués), de négociations ou consultations, et, dans certains cas, de conflits. En ce sens, les relations sociales au sein de l'entreprise accompagneraient plus qu'elles n'empêcheraient les réductions d'effectif ou de salaire. Seules les opinions prosyndicales des directions joueraient en sens contraire. On peut faire l'hypothèse que ces directions favorables au fait syndical mobilisent en priorité des formes d'ajustements pesant le moins possible sur les salariés (par exemple en utilisant leur trésorerie ou en réduisant les marges).

Le modèle III permet de tester quelques hypothèses complémentaires en comparant les relations sociales dans les établissements ayant procédé à des ajustements selon qu'il y a, ou non, un syndicat. Dans les deux pays, les caractéristiques sont proches, ce qui montre que la présence syndicale ne modifie pas fondamentalement l'état des relations sociales dans les établissements qui ont réduit leurs effectifs et/ou les salaires (par rapport à ceux qui ne l'ont pas fait). On peut simplement noter qu'en France, en présence de syndicats, les établissements négocient légèrement moins sur l'emploi (odds ratio passant de 1,36 à 1,22; tableau 4, modèle III) et qu'ils sont légèrement plus conflictuels (odds ratio passant de 1,28 à 1,62). La situation est différente en Grande-Bretagne: la présence syndicale va de pair avec des établissements qui, quand ils procèdent à des ajustements, consultent plus sur l'emploi (odds ratio passant 2,58 à 5,92 ) et tendent à devenir plus neutres à l'égard des syndicats (odds ratio passant de 0,29 à $0,69^{19}$ ). Ainsi, en cohérence avec la présentation habituelle des contextes institutionnels français et britannique, il semble que la présence syndicale s'accompagne d'orientations opposées en cas d'ajustement de l'emploi ou des salaires dans l'établissement, avec légèrement plus de conflictualité en France et de consultation en Grande-Bretagne. Ces résultats peuvent

18 Cependant, l'odds ratio correspondant à la situation britannique n'est pas significativement différent de 1, ce qui peut s'expliquer par la rareté des conflits collectifs en Grande-Bretagne (et leur association forte avec la présence syndicale).

${ }^{19}$ Ce résultat nous semble témoigner du fait que les ajustements sont plus spécifiquement le fait de directions antisyndicales quand il n'y a pas de syndicat. 
témoigner des marges de manœuvre des syndicats dans les deux pays: la négociation étant fortement encadrée par la loi en France, c'est principalement dans l'institution d'un rapport de force favorable que se situe une partie du répertoire de l'action syndicale (même si l'écart de conflictualité observé demeure faible). À l'opposé, dans le système volontariste britannique, parvenir à être consulté, et parfois à négocier, est déjà le signe d'un rapport de force plus favorable aux salariés. Quoi qu'il en soit, dans les deux pays, les syndicats apparaissent plus comme des institutions négociant les modalités d'ajustement, parfois au prix de conflits, que comme des obstacles à même de les empêcher.

\section{Ajustements internes versus externes: le rôle des relations sociales}

Le tableau 5 fournit les résultats des régressions multinomiales non ordonnées: la variable dépendante indique, parmi les établissements ayant procédé à des ajustements, s'il s'agit d'ajustements par l'emploi uniquement ou par l'emploi et les salaires - un ajustement par les salaires uniquement étant mis en référence. Ce tableau fait apparaître une différence nette entre les profils de relations sociales des établissements français et britanniques ayant opéré des ajustements externes (par une réduction des effectifs) plutôt qu'internes (par les salaires uniquement). En France, les ajustements par les effectifs sont plus le fait des établissements syndiqués, alors que c'est l'inverse de l'autre côté de la Manche. Certes les résultats statistiques ne sont pas significatifs pour la Grande-Bretagne dans le modèle IV (où les variables indicatrices des relations sociales sont limitées à la seule présence syndicale), mais ils le deviennent dans le modèle $\mathrm{V}$, qui est plus complet.

De ce dernier modèle, il ressort qu'en France la présence de syndicats ou de salariés syndiqués, la tenue de négociation sur l'emploi et une opinion prosyndicale de la direction caractérisent les établissements ayant mené une réduction des effectifs plutôt que des salaires uniquement. Une interprétation possible de ces résultats serait l'existence d'un accord, éventuellement tacite, des syndicats et des directions d'établissement pour garantir les salaires alors même que des réductions d'emploi ont lieu et font le plus souvent l'objet de négociations. Au-delà des objectifs affichés et traditionnels des syndicats en termes de rémunérations, on peut faire l'hypothèse que le maintien d'une dynamique des salaires résulte également d'une volonté des employeurs de maintenir la motivation des salariés, et ce dans un contexte d'insatisfaction salariale élevée (Askenazy, Bozio et García-Peñalosa, 2013). Loin du consensus national qui a prévalu dans les premiers mois suivant la crise, et dans un contexte d'inflation faible (qui limite la possibilité d'ajustements des salaires réels, ajustements qui ne peuvent dès lors qu'essentiellement porter sur les primes et parts variables), ce serait bien sur l'emploi qu'auraient principalement porté les ajustements en France. Cette interprétation apparaît cohérente avec l'évolution des salaires et de l'emploi observée au niveau macroéconomique, caractérisée par la poursuite d'une tendance à la hausse des salaires jusqu'en 2010 (ibid.). 
Tableau 5. Relation entre la présence syndicale seule, puis associée aux autres variables relatives aux relations sociales, sans interaction, et les modes d'ajustements (modèles IV et V)

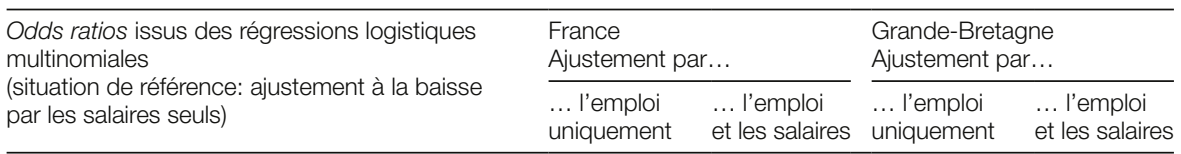

Modèle IV, présence syndicale (et variables de contrôle)

Présence d'un représentant syndical/

d'au moins un salarié syndiqué

$1,50 * * * \quad 2,09 * * * \quad 0,72$

Modèle V, présence syndicale et autres variables relatives aux relations sociales, sans interaction (et variables de contrôle)

\begin{tabular}{|c|c|c|c|c|}
\hline Présence syndicale & 1,37 & $2,02 * * *$ & $0,45^{*}$ & $0,55 * *$ \\
\hline Négociation/consultation sur l'emploi & $1,37 *$ & 1,21 & $10,1 * * *$ & $15,0 * * *$ \\
\hline $\begin{array}{l}\text { Négociation/négociation ou consultation } \\
\text { sur les salaires }\end{array}$ & 0,91 & 0,84 & $2,70 * * *$ & $2,56 * *$ \\
\hline Conflit/conflits ou menace de conflit & 0,96 & 0,81 & $2,81 *$ & $3,31 * *$ \\
\hline $\begin{array}{l}\text { Syndicats irremplaçables/en faveur } \\
\text { de la syndicalisation des salariés }\end{array}$ & $3,67 * * *$ & 1,33 & 0,74 & 0,59 \\
\hline
\end{tabular}

* Statistiquement significatif au seuil de 10 pour cent. ** Statistiquement significatif au seuil de 5 pour cent. *** Statistiquement significatif au seuil de 1 pour cent.

Champ: Établissements de 11 salariés et plus du secteur marchand non agricole.

Note: Données pondérées (poids établissement). Modèles: régressions logistiques multinomiales non ordonnées avec, comme variable dépendante, les modes d'ajustement à la baisse par l'emploi uniquement ou par l'emploi et les salaires conjointement (la situation de référence étant l'ajustement à la baisse par les salaires uniquement) et, comme variables indépendantes, outre les variables relatives aux relations sociales, la variable rendant compte d'un choc économique et des variables de contrôle relatives aux caractéristiques structurelles (non indiquées dans le tableau, mais l'ensemble des résultats du modèle IV, peu différents de ceux du modèle V, sont présentés dans le tableau A2 en annexe).

Source: Calculs des auteurs à partir des données des enquêtes REPONSE 2010-2011 et WERS 2011.

En Grande-Bretagne, la situation est différente. Certes, les ajustements par les effectifs sont associés à davantage de négociations et consultations et de conflits collectifs, signe de l'enjeu social que de telles opérations représentent, mais la présence de syndicats et les opinions prosyndicales sont, à l'inverse de la France, plus fréquentes dans les établissements ayant seulement réduit les salaires (plutôt que l'emploi). Reste que les négociations, notamment salariales, sont particulièrement peu fréquentes dans le cas d'ajustements par les salaires. L'ajustement par les salaires serait donc paradoxalement plus fréquent en présence de syndicats, mais moins souvent négocié, ce qui évoque là encore la présence d'accords tacites entre employeurs et salariés plutôt que de véritables négociations ou discussions.

\section{Discussion des résultats empiriques}

Le premier résultat mis en évidence est le fait que la mise en œuvre d'ajustements par la flexibilité interne ou externe de la part des entreprises va de pair avec des relations sociales actives, c'est-à-dire la présence de syndicats, de négociations et de conflits. Ce résultat fait écho à une intensification de la 
négociation pendant la crise que l'on a pu constater en France (Béthoux et coll., 2015), en Allemagne (Bellmann et Gerner, 2012; Zagelmeyer, 2016; Zagelmeyer, Heckmann et Kettner, 2012) et plus largement en Europe (Glassner, Keune et Marginson, 2011).

Deux interprétations différentes sont possibles: soit les relations sociales n'ont pas empêché les ajustements, soit les ajustements ont été contenus grâce à l'activité syndicale. Économétriquement, nous ne pouvons trancher entre ces deux lectures mais des travaux de terrain menés sur la période étayent plutôt l'hypothèse d'une impossibilité pour les syndicats à faire barrage aux ajustements (Perez et coll., 2015). De fait, la montée des négociations de compromis, qui a débuté bien avant la crise, favorise plutôt ce qu'on peut considérer comme une gestion conjointe, par les syndicats et les employeurs, des conséquences de la crise. Roche, Teague et Coughlan (2015) distinguent différentes formes de négociations de compromis, plus ou moins favorables à l'une des parties, employeur ou salariés, pour souligner que la crise a plutôt favorisé le développement des formes intermédiaires, celles de négociations de compromis «distributives» marquées par des concessions bilatérales. Si leur constat porte sur l'Irlande, il fait écho aux analyses proposées pour le cas allemand (Bellmann et Gerner, 2012; Zagelmeyer, 2016) et pourrait s'appliquer aux situations française ou britannique. Donnant une lecture un peu plus pessimiste des marges d'action syndicale, Perez et ses coauteurs (2015) soulignent que la conjoncture économique dégradée a pu peser sur les négociations et a même pu être utilisée par les employeurs pour imposer des compromis là où les conséquences de la crise n'étaient pas forcément prégnantes. Dans le cas du Portugal, Távora et González (2016) vont jusqu’à observer des conséquences de long terme du contexte de crise sur le fonctionnement de la négociation collective.

Un autre résultat clé de nos analyses est le poids des négociations portant sur l'emploi. Elles paraissent centrales dans la mise en place d'ajustements du volume d'emploi, alors que la négociation salariale n'est pas clairement associée aux ajustements salariaux (qui auraient lieu hors du cadre d'une négociation $^{20}$ ). En France, cela tient partiellement à des questions de procédure: les négociations accompagnent nécessairement la mise en œuvre des «plans de sauvegarde de l'emploi», dispositifs qui visent à éviter ou limiter les licenciements pour motif économique et à favoriser le reclassement des salariés licenciés, obligatoires pour les entreprises de plus de 50 salariés, en application du Code du travail. Mais le contenu de négociations sur l'emploi peut être plus large. Dans le cas français, une enquête de terrain récente (Perez et coll., 2015) révèle que les syndicats acceptent d'autant plus facilement les ajustements de l'emploi qu'ils se concentrent sur les contrats temporaires. Plus

${ }^{20}$ Par exemple par la limitation des primes et parts variables, qui se sont fortement développées en France au cours des dernières décennies (Demailly et Toutlemonde, 2014), et dont l'octroi se situe fréquemment hors du périmètre de la négociation salariale. 
généralement, derrière le rôle apparemment central des négociations sur l'emploi, il est nécessaire de se poser la question de leur contenu. Ainsi, Béthoux et ses coauteurs (2015) montrent que l'intensité du dialogue social peut cacher des échanges plus formels que réels. On retrouve le risque d'«évidement» des négociations, pratiques visant à limiter ce qui se joue dans le cadre des échanges, souligné par Giraud et Ponge (2016).

Une autre façon de considérer nos résultats empiriques consiste à s'interroger sur le rôle plus limité des négociations salariales dans la réaction à la crise, que ce soit par la mobilisation plus faible des ajustements salariaux lorsque des syndicats sont présents, en France, ou la moindre négociation d'ajustements salariaux alors qu'ils existent, en Grande-Bretagne. In fine, dans les deux cas, l'ajustement par les salaires serait peu soutenu par les syndicats, directement (via la présence syndicale) ou indirectement (via les négociations en Grande-Bretagne). Ce résultat fait écho à celui d'Amossé, Bryson et Petit (2016) qui montrent que, en France comme en Grande-Bretagne, la présence syndicale (associée à la reconnaissance syndicale dans le contexte britannique) avant la crise, en 2004-2005, est associée à moins de diminutions ou de gels salariaux ensuite. Comme nous avons pu l'évoquer plus haut, ces résultats peuvent illustrer le fait que les ajustements salariaux, lorsqu'ils existent, se passent de façon implicite plutôt qu'explicite. On peut en effet faire l'hypothèse que, si des ajustements par les salaires ont eu lieu en Grande-Bretagne, c'est d'abord grâce à la forte inflation britannique des années 2008 à 2010 qui a permis des ajustements salariaux sans révision des salaires nominaux de base. Une telle hypothèse paraît étayée par la prise en compte d'une autre variable de l'enquête WERS. En effet, si près de 40 pour cent des établissements indiquent avoir gelé ou réduit les salaires pour faire face à la crise (variable que nous étudions ici), une autre variable montre que moins de 1 pour cent des établissements ont déclaré avoir réduit les salaires lors de la dernière révision salariale de la principale catégorie de salariés de l'établissement. Si les syndicats britanniques n'apparaissent pas opposés à un ajustement salarial contrairement aux français, ce serait bien plus la conséquence d'un décalage d'inflation entre les deux pays que d'un positionnement syndical en faveur de baisses de salaires. Au-delà, si les syndicats britanniques ont peu négocié sur les salaires, ils n'ont pas davantage fait grève et on peut faire l'hypothèse qu'un tel ajustement a fait l'objet d'un accord tacite.

\section{Conclusion}

Malgré des tissus productifs différents, une exposition à la crise différenciée et des institutions souvent présentées comme opposées, les relations sociales caractérisant les établissements français et britanniques ayant procédé à des ajustements suite à la Grande Récession apparaissent étonnamment proches. Ces relations sociales prennent la forme, toutes choses égales par ailleurs, d'une plus grande présence syndicale, de négociations ou consultations plus fréquentes sur l'emploi que sur les salaires et de conflits collectifs plus 
probables. Ainsi, des relations sociales ouvertes accompagneraient les ajustements face à la crise, plutôt que de leur faire obstacle. Au vu des données, seules les opinions prosyndicales des directions d'entreprise permettraient de limiter la survenue de tels ajustements, ce qui pourrait être le signe d'une volonté de (ou de la capacité à) ne pas faire porter sur les salariés les conséquences de la crise.

Les principales différences entre les deux pays sont de deux ordres. Premièrement, la présence syndicale s'accompagnerait, en cas d'ajustement, d'une ambiance plus conflictuelle en France et plus favorable à la négociation en Grande-Bretagne. Ces différences, d'ampleur limitée et qui mériteraient donc d'être validées par d'autres travaux, témoignent de registres d'action différents de part et d'autre de la Manche. Deuxièmement, et principalement, si la présence syndicale et les opinions prosyndicales des directions font système dans les deux pays, les liens avec les modes d'ajustement opérés sont de sens opposé: en France, ce sont davantage les établissements ayant réduit leurs effectifs (ajustements externes) qui ont des syndicats et des directions prosyndicales, alors que ce sont les établissements ayant déclaré avoir gelé ou baissé leurs salaires qui sont dans cette situation en Grande-Bretagne. Cette différence renvoie à la possibilité ouverte, par la politique monétaire britannique, d'ajuster les salaires réels de façon discrète, ce qui n'a pas été le cas en France. Si cette interprétation est juste, cela pourrait donc signifier que les deux pays se distingueraient en premier lieu par des politiques macroéconomiques différentes, notamment monétaires, plutôt que par le rôle supposé des institutions du marché du travail.

Dans l'ensemble, ces résultats vont à l'encontre de l'hypothèse selon laquelle les syndicats constituent des entraves à la flexibilité des salaires ou de l'emploi, et ils contrastent avec la présentation traditionnelle des systèmes de relations professionnelles français et britannique. Ils ouvrent de nouveaux questionnements: sont-ils le signe d'une convergence vers un modèle de relations sociales en entreprise tourné vers un objectif de flexibilité et/ou le signe d'une faiblesse accrue des syndicats? Et, plus généralement, témoignent-ils d'un affaiblissement global des spécificités nationales des relations professionnelles (Meardi, 2012; Colvin et Darbishire, 2013)? Ou bien sont-ils simplement le résultat de réactions particulières dans le contexte de la crise de 2007-2009 (Hyman, 2010)?

\section{Références}

Amable, B. 2003. The diversity of modern capitalism. Oxford, Oxford University Press.

Amossé, T.; Bryson, A.; Forth, J.; Petit, H. (dir. de publ.). 2016. Comparative workplace employment relations: An analysis of practice in Britain and France. Londres, Palgrave Macmillan.

-; -; Petit, H. 2016. «How did workplaces respond to recession?», dans l'ouvrage publié sous la direction de T. Amossé, A. Bryson, J. Forth et H. Petit: Comparative workplace employment relations: An analysis of practice in Britain and France, pp. 211-237. Londres, Palgrave Macmillan. 
-; Coutrot, T. 2008. «Genèse et réalité d'une enquête», dans l'ouvrage publié sous la direction de T. Amossé, C. Bloch-London et L. Wolff: Les relations sociales en entreprise: un portrait à partir des enquêtes "Relations professionnelles et négociations d'entreprise [REPONSE 1992-1993, 1998-1999 et 2004-2005], pp. 38-68. Paris, La Découverte.

-; Forth, J. 2016. «Employee expression and representation at work: Voice or exit?», dans l'ouvrage publié sous la direction de T. Amossé, A. Bryson, J. Forth et H. Petit: Comparative workplace employment relations: An analysis of practice in Britain and France, pp. 61-99. Londres, Palgrave Macmillan.

Askenazy, P.; Bozio, A.; García-Peñalosa, C. 2013. «Dynamique des salaires par temps de crise», Notes du conseil d'analyse économique, vol. 5, $\mathrm{n}^{\circ}$ 5, pp. 1-12.

Bellmann L. Gerner, H.-D. 2012. «Company-level pacts for employment in the global crisis 2008/2009: First evidence from representative German establishment-level panel data», International Journal of Human Resource Management, vol. 23, $\mathrm{n}^{\circ}$ 16, pp. 3375-3396.

-; -; Laible, M.-C. 2016. «The German labour market puzzle in the Great Recession», dans l'ouvrage publié sous la direction de P. Askenazy, L. Bellmann, A. Bryson et E. Moreno Galbis: Productivity puzzles across Europe, pp. 187-235. Oxford, Oxford University Press.

Béthoux, E.; Mias, A.; Blache, G.; Dupuy, C.; Jobert, A.; Renoux, J.-L.; Spieser, C.; Tallard, M.; Vincent, C. 2015. «Dialoguer plus, mais sur quoi? Les régulations d'entreprise en matière d'emploi, de formation et de conditions de travail en temps de crise», La Revue de l'IRES, $\mathrm{n}^{\circ}$ 84, 2015/1, pp. 91-123.

Bevort, A.; Jobert, A. 2011. Sociologie du travail: les relations professionnelles, $2^{e}$ édition. Paris, Armand Colin.

Bloch-London, C.; Pélisse, J. 2008. «L'évolution du cadre légal des relations professionnelles: entre foisonnement juridique et renouvellement des acteurs, une appropriation sélective des dispositifs», dans l'ouvrage publié sous la direction de T. Amossé, C. Bloch-London et L. Wolff: Les relations sociales en entreprise: un portrait à partir des enquêtes «Relations professionnelles et négociations d'entreprise» [REPONSE 1992-1993, 1998-1999 et 2004-2005], pp. 102-122. Paris, La Découverte.

Brown, W.; Bryson, A.; Forth, J. 2009. «Competition and the retreat from collective bargaining», dans l'ouvrage publié sous la direction de W. Brown, A. Bryson, J. Forth et $\mathrm{K}$. Whitfield: The evolution of the modern workplace, pp. 22-47. Cambridge, Cambridge University Press.

Bryson, A.; Frege, C. 2010. «The importance of comparative workplace employment relations studies», British Journal of Industrial Relations, vol. 48, n 2, pp. 231-234.

-; Forth, J. 2016. «The UK's productivity puzzle», dans l'ouvrage publié sous la direction de P. Askenazy, L. Bellmann, A. Bryson et E. Moreno Galbis: Productivity puzzles across Europe, pp. 129-173. Oxford, Oxford University Press.

-; -; Laroche, P. 2011. «Evolution or revolution? The impact of unions on workplace performance in Britain and France», European Journal of Industrial Relations, vol. 17, $\mathrm{n}^{\circ}$ 2, pp. 171-187.

Caroli, E.; Gautié, J.; Lloyd, C.; Lamanthe, A.; James, S. 2010. «Delivering flexibility: Contrasting patterns in the French and the UK food processing industry», British Journal of Industrial Relations, vol. 48, $\mathrm{n}^{\circ} 2$, pp. 284-309.

-; Van Reenen, J. 2001. «Skill biased organizational change? Evidence from a panel of British and French establishments», Quarterly Journal of Economics, vol. 116, $\mathrm{n}^{\circ} 4$, pp. 1449-1492.

Colvin, A. J. S.; Darbishire, O. 2013. «Convergence in industrial relations institutions: The emerging Anglo-American model?», Industrial and Labor Relations Review, vol. 66, $\mathrm{n}^{\circ} 5$, pp. $1047-1077$.

Commission européenne. 2011. Industrial relations in Europe 2010, Direction générale de l'emploi, des affaires sociales et de l'inclusion. Luxembourg, Office des publications de l'Union européenne. 
Conway, N.; Deakin, S.; Konzelmann, S.; Petit, H.; Rebérioux, A.; Wilkinson, F. 2008. «The influence of stock market listing on human resource management: Evidence for France and Britain», British Journal of Industrial Relations, vol. 46, n' ${ }^{\circ}$, pp. 631-673.

Coutrot, T. 1998. «How do institutional frameworks affect industrial relations outcomes? A micro-statistical comparison of France and Britain», European Journal of Industrial Relations, vol. 4, $\mathrm{n}^{\circ}$ 2, pp. 177-205.

Crouch, C. 1993. Industrial relations and European state traditions. Oxford, Clarendon Press.

Delahaie, N.; Vincent, C. 2016. «Négociations salariales en Europe: dynamiques institutionnelles et stratégies d'acteurs», La Revue de l'IRES, n 89, 2016/2, pp. 27-50.

Demailly, D.; Toutlemonde, F. 2014. «Les pratiques salariales des entreprises», Dares Analyses, $\mathrm{n}^{\circ}$ 092. Paris, Dares.

Dickens, L.; Hall, M. 2003. «Labour law and industrial relations: A new settlement?», dans l'ouvrage publié sous la direction de P. Edwards: Industrial relations: Theory and practice, deuxième édition, pp. 124-156. Oxford, Blackwell Publishing.

Freyssinet, J. 2016. Les bonnes pratiques de dialogue social en période de sortie de crise dans l'Union européenne: le cas de la France, document publié dans le cadre d'un projet financé par l'UE, et géré par le Département de la gouvernance et du tripartisme en collaboration avec le Centre international de formation de l'OIT, disponible à l'adresse https://www.ilo.org/wcmsp5/groups/public/---europe/---ro-geneva/---ilo-paris/ documents/publication/wcms_484113.pdf [consulté le 3 juillet 2019].

Giraud, B.; Ponge, R. 2016. «Des négociations entravées: les ressorts ambivalents de l'institutionnalisation de la négociation collective en entreprise», La Nouvelle Revue du Travail [document mis en ligne le $1^{\text {er }}$ juin 2016, consulté le 17 juin 2019, disponible à l'adresse http://journals.openedition.org/nrt/2591].

Glassner, V.; Keune, M.; Marginson, P. 2011. «Collective bargaining in a time of crisis: Developments in the private sector in Europe», European Review of Labour and Research, vol. 17, $\mathrm{n}^{\circ}$ 3, pp. 303-322.

Hall, P. A.; Soskice, D. (dir. de publ.). 2001. Varieties of capitalism: The institutional foundations of comparative advantage. Oxford, Oxford University Press.

Hyman, R. 2010. Social dialogue and industrial relations during the economic crisis: Innovative practices or business as usual?, Working Paper No. 11. Genève, Département des relations professionnelles et des relations d'emploi, BIT.

Jobert, A. 2017. «Dynamique de la négociation collective décentralisée en France, entreprise et territoire», dans l'ouvrage publié sous la direction de C. Alessi et L. Guaglianone: Ragioni e passioni della contrattazione collettiva di secondo livello: tendenze italiane ed europee, pp. 49-68. Rome, Aracne Editrice.

-. 2000. Les espaces de la négociation collective, branches et territoires. Toulouse, Octarès.

Marginson, P. 2015. «The changing nature of collective employment relations», Employee Relations, vol. 37, $\mathrm{n}^{\circ}$ 6, pp. 645-657.

Marsden, D. 2013. «Individual voice in employment relationships: A comparison under different forms of workplace representation», Industrial Relations: A Journal of Economy and Society, vol. 52, $\mathrm{n}^{\mathrm{o}} \mathrm{S1}$, pp. 221-258.

-. 2010. The end of national models in employment relations?, CEP Discussion Papers No. 998. Londres, Centre for Economic Performance, London School of Economics and Political Science.

-; Belfield, R. 2010. «Institutions and the management of human resources: Incentive pay systems in France and Great Britain», British Journal of Industrial Relations, vol. $48, \mathrm{n}^{\circ} 2$, pp. $235-283$.

Meardi, G. 2012. «Industrial relations after European state traditions», dans l'ouvrage publié sous la direction de L. Burroni, M. Keune et G. Meardi: Economy and society in Europe: A relationship in crisis, pp. 100-123. Cheltenham, Edward Elgar Publishing.

Milner, S. 2015. Comparative employment relations: France, Germany and Britain. Londres, Palgrave Macmillan.

Perez, C.; Thèvenot, N.; Berta, N.; Brochard, D.; Delahaie, N.; Jallais, S.; Perraudin, C.; Sauviat, C.; Valentin, J. 2015. «Modes d'ajustement par le travail en temps de crise: des relations professionnelles sous tension», La Revue de l'IRES, n ${ }^{\circ}$ 4, 2015/1, pp. 61-90. 
Roche, W. K.; Teague, P.; Coughlan, A. 2015. «Employers, trade unions and concession bargaining in the Irish recession», Economic and Industrial Democracy, vol. 36, $\mathrm{n}^{\circ} 4$, pp. 653-676.

Távora, I.; González, P. 2016. «Labour market regulation and collective bargaining in Portugal during the crisis: Continuity and change», European Journal of Industrial Relations, vol. 22, $\mathrm{n}^{\circ} 3$, pp. 251-265.

van Wanrooy, B.; Bewley, H.; Bryson, A.; Forth, J.; Freeth, S.; Stokes, L.; Wood, S. 2013. The 2011 workplace employment relations study: First findings. Londres, Department for Business, Innovation and Skills.

Zagelmeyer, S. 2016. «Tackling the crisis through concession bargaining: Evidence from five German companies», International Journal of Manpower, vol. 34, $\mathrm{n}^{\circ} 3$, pp. 232-251.

-; Heckmann, M.; Kettner, A. 2012. «Management responses to the global financial crisis in Germany: Adjustment mechanisms at establishment level», International Journal of Human Resource Management, vol. 23, n 16, pp. 3355-3374. 


\section{Annexe \\ Les principales variables utilisées pour caractériser les relations sociales en France et en Grande-Bretagne}

Présence syndicale: De nombreuses variables sont utilisées dans l'enquête REPONSE, qui permettent d'identifier la présence de salariés syndiqués (TXSYND, TXSYNDT_10), d'un délégué syndical (DSETAB, DSENTR), d'un représentant de section syndicale (RSS) ou d'un représentant du personnel élu sur liste syndicale, quelles que soient l'instance (délégation du personnel, délégation unique, comité d'établissement ou d'entreprise, comité d'hygiène, de sécurité et des conditions de travail) et l'organisation syndicale $^{1}$; dans l'enquête WERS, les variables utilisées sont ZUNIONUM, ESTEWARD et EOTHUREP.

Existence de négociations ou consultations sur l'emploi et les salaires: Grâce aux variables NEGSL10-ADERNEG et EMPLOI de REPONSE, on identifie les établissements où les salaires et/ou l'emploi ont fait l'objet de négociations entre syndicat et direction au cours des trois dernières années. Dans WERS, les variables FCONS et $J R E D C O N 1 / J R E D C O N 2$ correspondent respectivement à l'existence d'une consultation ou d'une négociation avec les représentants du personnel à l'occasion de la dernière révision des salaires de la principale catégorie de salariés de l'établissement et à la consultation des salariés ou de leurs représentants concernant les licenciements économiques envisagés au cours des douze derniers mois (qu'ils aient ou non eu lieu).

Conflits collectifs (survenue de conflit collectif du travail au cours des trois dernières années (2008-2010) en France et survenue ou menace de tels conflits au cours des douze derniers mois en Grande-Bretagne): Les variables $D E B R A_{-}, G R E V 2_{-}, G R E V 3_{-}$, $P E R L E_{-}, Z E L E_{-}$et $H S U P_{-}$(relatives aux débrayages, grèves de plus ou de moins de deux jours, grèves perlées et du zèle et refus d'heures supplémentaires) pour REPONSE et GACT et GTHREAT (avec des modalités relatives aux grèves, grèves du zèle et refus d'heures supplémentaires) pour WERS ont été utilisées. La rareté des conflits collectifs en Grande-Bretagne - leur survenue au cours des douze derniers mois concerne moins de 1,6 pour cent des établissements britanniques du champ de notre étude en 2011-2012 (Amossé et Forth, 2016) - rend délicate l'utilisation de ce seul indicateur.

Opinion des directions d'entreprise à propos des syndicats: Dans REPONSE, la variable SYIRREM («les syndicats jouent un rôle irremplaçable dans la représentation des salariés») a été utilisée, avec la seule modalité «tout à fait d'accord». Pour WERS, c'est la variable EVIEWS (directions «favorables à la syndicalisation des salariés», plutôt qu'«indifférentes» ou «défavorables») qui a été utilisée.

${ }^{1}$ Le programme de construction de la variable est disponible sur demande auprès des auteurs. 
Tableau A1. Comparaison des tissus productifs en France et en Grande-Bretagne

\begin{tabular}{|c|c|c|}
\hline & France & Grand \\
\hline \multicolumn{3}{|c|}{$\begin{array}{l}\text { Caractéristiques des établissements } \\
\text { (en pourcentage de l'ensemble des établissements de l'échantillon) }\end{array}$} \\
\hline \multicolumn{3}{|l|}{ Ancienneté de l'établissement } \\
\hline Moins de 5 ans & 4,2 & 10,3 \\
\hline De 5 à 9 ans & 10,7 & 17,1 \\
\hline De 10 à 19 ans & 25,0 & 24,6 \\
\hline De 20 à 49 ans & 41,7 & 36,1 \\
\hline 50 ans et plus & 18,4 & 11,9 \\
\hline \multicolumn{3}{|l|}{ Taille de l'établissement } \\
\hline De 11 à 19 salariés & 38,0 & 46,2 \\
\hline De 20 à 49 salariés & 39,1 & 34,5 \\
\hline De 50 à 99 salariés & 13,3 & 10,9 \\
\hline De 100 à 199 salariés & 5,6 & 5,0 \\
\hline De 200 à 499 salariés & 3,0 & 2,6 \\
\hline 500 salariés et plus & 0,9 & 0,8 \\
\hline \multicolumn{3}{|l|}{ Activité principale } \\
\hline CDE Industrie & 17,6 & 10,6 \\
\hline F Construction & 11,1 & 5,6 \\
\hline G Commerce & 19,5 & 21,4 \\
\hline H Transports et entreposage & 7,7 & 4,7 \\
\hline I Hébergement et restauration & 6,4 & 12,4 \\
\hline JR Information, communication et spectacles & 4,0 & 5,6 \\
\hline KL Activités financières, d'assurance et immobilières & 4,2 & 4,6 \\
\hline M Activités spécialisées, scientifiques et techniques & 6,7 & 12,3 \\
\hline N Activités de services administratifs et de soutien & 5,1 & 4,2 \\
\hline PQ Enseignement, santé humaine et action sociale & 14,5 & 17,2 \\
\hline S Autres activités de services & 3,1 & 1,5 \\
\hline \multicolumn{3}{|l|}{ Structure de gouvernance et de capital } \\
\hline Établissement autonome & 40,8 & 41,9 \\
\hline Cotation directe ou indirecte en Bourse & 14,0 & 11,0 \\
\hline Capital à dominante familiale & 47,2 & 41,3 \\
\hline \multicolumn{3}{|l|}{ Dimension du marché } \\
\hline Marché local & 35,5 & 44,8 \\
\hline Marché régional & 22,3 & 16,0 \\
\hline Marché national & 24,0 & 26,2 \\
\hline Marché international & 18,2 & 13,1 \\
\hline
\end{tabular}

Composition de la main-d'œuvre au sein des établissements (valeurs moyennes)

$\begin{array}{lrr}\text { Pourcentage de salariés femmes } & 42,6 & 45,4 \\ \text { Pourcentage de salariés de } 21 \text { ans ou moins } & 2,9 & 8,5 \\ \text { Pourcentage de salariés de } 50 \text { ans ou plus } & 22,9 & 22,1 \\ \text { Pourcentage de cadres et d'ingénieurs } & 16,6 & 24,9 \\ \text { Pourcentage de techniciens et d'agents de maîtrise } & 19,7 & 9,7 \\ \text { Pourcentage d'employés de bureau et assimilés } & 29,3 & 35,8 \\ \text { Pourcentage d'ouvriers } & 34,4 & 29,6 \\ \text { Pourcentage de contrats à durée déterminée } & 10,4 & 7,2 \\ \text { Pourcentage d'intérimaires } & 3,8 & 3,5\end{array}$

Champ: Établissements de 11 salariés et plus du secteur marchand non agricole.

Note: Données pondérées (poids établissement pour l'ensemble des variables, à l'exception des variables relatives à la composition de la main-d'œuvre pour lesquelles les poids salariés sont utilisés). Les activités sont classées selon la Nomenclature d'activités française (NAF) rév. 2.

Source: Calculs des auteurs à partir des données des enquêtes REPONSE 2010-2011 et WERS 2011. 
Tableau A2. Présence syndicale et modes d'ajustements (modèle IV, résultats complets)

\begin{tabular}{|c|c|c|c|c|}
\hline \multirow{2}{*}{$\begin{array}{l}\text { Coefficients issus des régressions logistiques multinomiales } \\
\text { (situation de référence: ajustement à la baisse } \\
\text { par les salaires uniquement) }\end{array}$} & \multicolumn{2}{|c|}{$\begin{array}{l}\text { France } \\
\text { Ajustement par... }\end{array}$} & \multicolumn{2}{|c|}{$\begin{array}{l}\text { Grande-Bretagne } \\
\text { Ajustement par... }\end{array}$} \\
\hline & $\begin{array}{l}\text {... l'emploi } \\
\text { uniquement }\end{array}$ & $\begin{array}{l}\text {... l'emploi } \\
\text { et les salaires }\end{array}$ & $\begin{array}{l}\text {... l'emploi } \\
\text { uniquement }\end{array}$ & $\begin{array}{l}\text {... l'emploi } \\
\text { et les salaires }\end{array}$ \\
\hline \multicolumn{5}{|l|}{ Dynamique de l'activité } \\
\hline Stable ou à la hausse & Réf. & Réf. & Réf. & Réf. \\
\hline À la baisse & $1,30 * * *$ & $1,68 * * *$ & 0,13 & $1,54 * * *$ \\
\hline \multicolumn{5}{|l|}{ Ancienneté de l'établissement } \\
\hline Moins de 5 ans & $1,21 * *$ & 0,44 & 0,46 & $-0,33$ \\
\hline De 5 à 9 ans & 0,41 & 0,19 & 0,31 & $-0,13$ \\
\hline De 10 à 19 ans & Réf. & Réf. & Réf. & Réf. \\
\hline De 20 à 49 ans & $-0,03$ & $0,69 * *$ & 0,14 & $-0,40$ \\
\hline 50 ans et plus & 0,22 & 0,08 & 0,12 & 0,16 \\
\hline \multicolumn{5}{|l|}{ Taille de l'établissement } \\
\hline De 11 à 19 salariés & $-0,16$ & $0,81 *$ & $-1,49 * * *$ & $-1,81 * * *$ \\
\hline De 20 à 49 salariés & 0,06 & 0,29 & $-0,88 * *$ & $-0,81 * *$ \\
\hline De 50 à 99 salariés & Réf. & Réf. & Réf. & Réf. \\
\hline De 100 à 199 salariés & 0,36 & 0,54 & 0,66 & 0,32 \\
\hline De 200 à 499 salariés & 0,84 & 0,84 & 1,16 & 0,75 \\
\hline 500 salariés et plus & 1,31 & 0,97 & 1,36 & 0,40 \\
\hline \multicolumn{5}{|l|}{ Activité principale } \\
\hline CDE Industrie & 0,50 & 0,26 & 0,06 & 0,57 \\
\hline F Construction & 0,60 & $-0,12$ & 0,33 & $1,34 * * *$ \\
\hline G Commerce & Réf. & Réf. & Réf. & Réf. \\
\hline H Transports et entreposage & $0,77^{*}$ & 0,46 & 0,53 & 0,10 \\
\hline I Hébergement et restauration & 0,21 & 0,40 & $-0,45$ & $-1,86 * * *$ \\
\hline JR Information, communication et spectacles & 0,48 & $1,00 *$ & $-0,85$ & $-0,28$ \\
\hline L Activités financières et immobilières & $3,27 * * *$ & $2,77 * *$ & $-0,96$ & 0,09 \\
\hline M Activités spécialisées, scientifiques et techniques & 0,44 & 0,47 & $-0,59$ & $-0,21$ \\
\hline N Activités de services administratifs et de soutien & 0,15 & 0,12 & 0,23 & 0,08 \\
\hline PQ Enseignement, santé humaine et action sociale & 0,46 & $0,89 *$ & $-0,44$ & $-0,70$ \\
\hline S Autres activités de services & 4,66 & 4,99 & $-0,84$ & $-0,66$ \\
\hline \multicolumn{5}{|l|}{ Structure de gouvernance et de capital } \\
\hline Établissement autonome & 0,16 & 0,35 & $-0,47 * *$ & $-0,13$ \\
\hline Cotation directe ou indirecte en Bourse & 0,30 & $-0,64 *$ & $-0,15$ & $-0,30$ \\
\hline Capital à dominante familiale & 0,01 & $0,49 *$ & $-0,87 * * *$ & $-0,48 * *$ \\
\hline \multicolumn{5}{|l|}{ Dimension du marché } \\
\hline Marché local & Réf. & Réf. & Réf. & Réf. \\
\hline Marché régional & 0,29 & 0,45 & 0,02 & $-0,02$ \\
\hline Marché national & 0,26 & $0,89 * * *$ & $0,81 * * *$ & 0,03 \\
\hline Marché international & 0,20 & $1,68 * * *$ & $1,00 * * *$ & 0,51 \\
\hline
\end{tabular}


Tableau A2. Présence syndicale et modes d'ajustements (modèle IV, résultats complets) (suite et fin)

Coefficients issus des régressions logistiques multinomiales

France

Grande-Bretagne

(situation de référence: ajustement à la baisse

par les salaires uniquement)

Ajustement par...

Ajustement par..

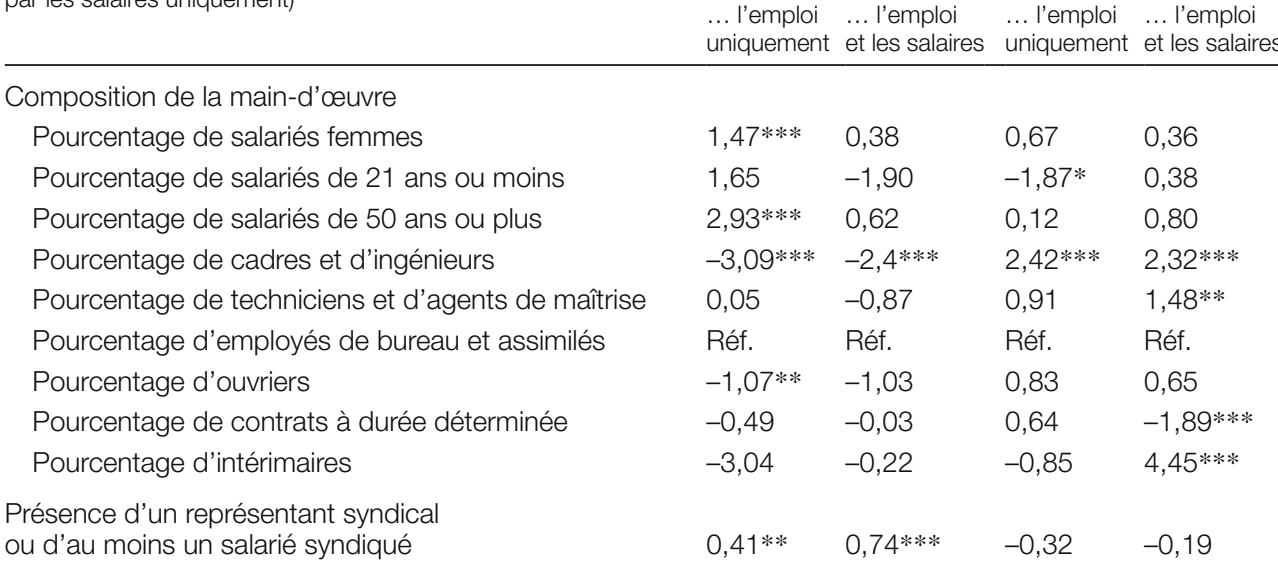

* Statistiquement significatif au seuil de 10 pour cent. *** Statistiquement significatif au seuil de 5 pour cent. *** Statistiquement significatif au seuil de 1 pour cent.

Champ: Établissements de 11 salariés et plus du secteur marchand non agricole.

Note: Données pondérées (poids établissement pour l'ensemble des variables, à l'exception des variables relatives à la composition de la main-d'œuvre pour lesquelles les poids salariés sont utilisés). Modèles: régressions logistiques multinomiales non ordonnées avec, comme variable dépendante, les modes d'ajustement à la baisse par l'emploi uniquement ou par l'emploi et les salaires conjointement (la situation de référence étant l'ajustement à la baisse par les salaires seuls) et, comme variables indépendantes, outre les variables relatives aux relations sociales, une variable rendant compte du choc économique et des variables de contrôle relatives aux caractéristiques structurelles. Les activités sont classées selon la Nomenclature d'activités française (NAF) rév. 2.

Source: Calculs des auteurs à partir des données des enquêtes REPONSE 2010-2011 et WERS 2011. 